Research Article

\title{
Classical Flutter and Active Control of Wind Turbine Blade Based on Piezoelectric Actuation
}

\author{
Tingrui Liu \\ College of Mechanical \& Electronic Engineering, Shandong University of Science \& Technology, Qingdao 266590, China \\ Correspondence should be addressed to Tingrui Liu; liutingrui9999@163.com
}

Received 18 April 2015; Revised 7 July 2015; Accepted 7 July 2015

Academic Editor: Mario Terzo

Copyright (C) 2015 Tingrui Liu. This is an open access article distributed under the Creative Commons Attribution License, which permits unrestricted use, distribution, and reproduction in any medium, provided the original work is properly cited.

\begin{abstract}
The aim of this article is to analyze classical flutter and active control of single-cell thin-walled composite wind turbine blade beam based on piezoelectric actuation. Effects of piezoelectric actuation for classical flutter suppression on wind turbine blade beam subjected to combined transverse shear deformation, warping restraint effect, and secondary warping are investigated. The extended Hamilton's principle is used to set up the equations of motion, and the Galerkin method is applied to reduce the aeroelastic coupled equations into a state-space form. Active control is developed to enhance the vibrational behavior and dynamic response to classical aerodynamic excitation and stabilize structures that might be damaged in the absence of control. Active optimal control scheme based on linear quadratic Gaussian (LQG) controller is implemented. The research provides a way for rare study of classical flutter suppression and active control of wind turbine blade based on piezoelectric actuation.
\end{abstract}

\section{Introduction}

Although instability of stall nonlinear flutter has generally been an important issue in flutter research [1], classical flutter was observed involving a high-speed rotating blade in pitch excitation process. Simultaneously, with the advent of large wind turbine fitted with relatively slender blades, classical flutter may become a more important design consideration. In addition, innovative blade designs involving the use of aeroelastic tailoring and structural tailoring, wherein the blade twists as it bends under the action of aerodynamic loads to shed load resulting from wind turbulence, may increase the blade's proclivity for flutter [2].

Most of the literature of classical flutter focused on the helicopter blades, noncoupling wing sections, and fan rotor blades [3-5]. As for active control, most of the literature focused on beam structures rather than the blade sectional shapes. Kapuria and Yasin [6] study the active vibration suppression of hybrid composite and fiber metal laminate plates integrated with piezoelectric fiber reinforced composite sensors and actuators. Phung-Van et al. [7] present an effective formulation based on higher-order shear deformation theory to investigate free vibration and dynamic control of piezoelectric composite plates integrated with sensors and actuators. Supersonic flutter control of a three-layered sandwich curved panel of rectangular plan form with an adaptive electrorheological fluid core layer is investigated by Hasheminejad and Motaaleghi [8].

Park and Kim [9] study the active twist rotor blade incorporating single crystal macrofiber composite actuators and analyze the aeroelasticity. Although the rotor blade properties dynamically represent a real rotor blade, the analytical objects, are the torsional behavior of helicopter blades or the vibration behavior of airfoils in which only the torsional motions are involved. Simultaneously, the objects of the existing literature mostly concentrate on the frequency research rather than time response analysis.

In this work the classical flutter and flutter suppression of composite blade beam are investigated for single-cell thinwalled structure with piezoelectric patch embedded. The validity of the piezoelectric actuation is tested and illustrated by time domain response analysis rather than frequency research. The analysis is applied to a laminated host structure of the circumferentially asymmetric stiffness (CAS) that produces bending-twist-transverse shear coupling. The governing system can be derived by the extended Hamilton principle. The spanwise distributed PZT-4 sensor/actuator pair is embedded into the orthotropic host. The net voltage 


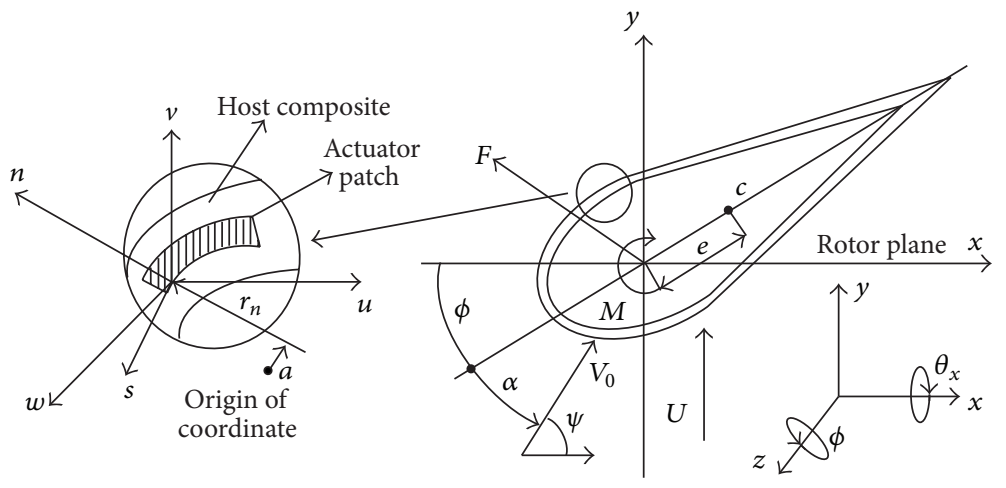

FIgURE 1: Coordinate system and aerodynamics and structure.

output from sensor is fed to a controller for the purpose of actuation. For piezoelectric actuation, active feedback control law and linear quadratic Gaussian controller are implemented. The purpose of present study is to investigate the validity of piezoelectric actuation under extreme conditions especially in critical region.

\section{Analytical Model and Equations of Motion}

Consider the thin-walled structure in which piezoelectric patch is embedded as Figure 1. The length $L$ of the blade is along $z$ direction. The origin of the rotating axis system is located at the rigid root in which the blade beam is mounted. $\Omega$ is the rotating speed; $c$ is the chord length; $h$ is the thickness of section; $r_{n}$ is the radius of curvature of the middle surface; $\phi$ is the twist angle of section; $U$ is the wind velocity. It is assumed that $c \ll L, h \ll c$, and $h \ll r_{n}$. The equation of the middle line of the closed section is as follows [2]:

$$
\begin{aligned}
y & =e\left\{\exp \left[0.05\left(1-\cos \theta_{\varphi}\right)^{2}+0.05\left(\sin \theta_{\varphi}\right)^{2}\right]\right. \\
& +\exp \left[-0.05\left(1-\cos \theta_{\varphi}\right)^{2}-0.05\left(\sin \theta_{\varphi}\right)^{2}\right] \\
& \left.\cdot \cos \theta_{\varphi}\right\},
\end{aligned}
$$

$$
\begin{aligned}
z= & e\left\{\exp \left[0.05\left(1-\cos \theta_{\varphi}\right)^{2}+0.05\left(\sin \theta_{\varphi}\right)^{2}\right]\right. \\
& -\exp \left[-0.05\left(1-\cos \theta_{\varphi}\right)^{2}-0.05\left(\sin \theta_{\varphi}\right)^{2}\right] \\
& \left.\cdot \sin \theta_{\varphi}\right\},
\end{aligned}
$$

where $e$ is the $1 / 4$ chord length and $\theta_{\varphi}$ changes from $0 \sim 2 \pi$.

The configuration of the piezoelectric actuator is assumed to be distributed over the entire blade span. The characteristics of the host composite structure and piezoelectric structure are depicted as (a) transverse shear, warping restraint effect, and secondary warping as depicted in [10]; (b) the master structure consists of 6-layer CAS configuration; the actuator is 1 piezoelectric layer, with the height $b_{k}^{a}$ and width $s_{k}^{a}$ along the circumferential $s$, spanwise $z$, and transverse $n$ directions; the width of piezoelectric layer $s_{k}^{a}$ is relatively small, generally less than or equal to $1 / 6$ chord length; (c) the piezoelectric elements may be employed concurrently for sensing and actuation and relate the voltage produced by the piezoelectric layer to the strain in the host structure; (d) the feedback control is achieved through the action of piezoelectrically induced vertical transverse shear motion at the blade tip.

To simplify the analysis, a structural tailoring technology for symmetric thin-walled structure is applied [10]. Consider the cantilever vibration first, ignore rotation about $y$, and retain rotation about $x$ according to the requirements of piezoelectric actuation, the displacements $u, v, w, \theta_{x}$, and $\phi$ are displayed in Figure 1, and the system kinetic energy $T$ and potential energy $V$ are, respectively, expressed as

$$
T=\frac{1}{2} \int_{0}^{L} \oint \sum_{k=1}^{N} \int_{h(k)} \rho^{(k)}\left\{(\dot{u}-y \dot{\phi})^{2}+(\dot{v}+x \dot{\phi})^{2}+\left[\left(\dot{W}+y \dot{\theta}_{x}-F_{w} \dot{\phi}^{\prime}\right)-\left(\frac{d x}{d s} \dot{\theta}_{x}+a \dot{\phi}^{\prime}\right)\right]^{2}\right\} d n d s d z
$$

where the primary warping function $F_{w}$ is depicted as

$$
F_{w}=\int_{0}^{s}\left[r_{n}(s)-2 \frac{A_{e}}{\beta}\right] d s,
$$

where $A_{e}$ denotes the section area bounded by the midline and $\beta$ denotes the length of the contour midline. The radius of curvature of the middle surface is expressed as 
TABLE 1: Composite parameters and piezoelectric properties.

\begin{tabular}{|c|c|c|c|}
\hline Composite items & Values & Piezoelectric items & Values \\
\hline Maximum exterior width & $24.21 \times 10^{-3} \mathrm{~m}$ & Thickness $t^{a}$ & $1.9 \times 10^{-4} \mathrm{~m}$ \\
\hline Maximum exterior height & $2.643 \times 10^{-3} \mathrm{~m}$ & Piezoelectric coefficient $e_{31}$ & $-2.05 \times 10^{2} \mathrm{PaV}$ \\
\hline Blade density $\rho_{m}$ & $1672 \mathrm{~kg} / \mathrm{m}^{3}$ & Density & $7.65 \times 10^{2} \mathrm{~kg} \mathrm{~s}^{2} / \mathrm{m}^{4}$ \\
\hline Ply layers & 6 & Electrical permittivity $\xi_{3}{ }^{p}$ & $1.2 \times 10^{-8} \mathrm{~F} / \mathrm{m}$ \\
\hline Ply thickness & $127 \times 10^{-6} \mathrm{~m}$ & Elastic coefficient $C_{11}$ & $1.39 \times 10^{11} \mathrm{~Pa}$ \\
\hline Ply layers & 6 & Liastic coemitertit & \\
\hline$G_{12} / v_{12}$ & $3.5 \mathrm{GPa} / 0.34$ & Elastic coefficient $C_{12}$ & $7.778 \times 10^{10} \mathrm{~Pa}$ \\
\hline$E_{11} / E_{22}$ & $25.8 \mathrm{GPa} / 8.7 \mathrm{GPa}$ & Piezoelectric width $s_{k}{ }^{a}$ & $\mathrm{c} / 6 \mathrm{~m}$ \\
\hline
\end{tabular}

$$
\begin{aligned}
r_{n} & =x \frac{d y}{d s}-y \frac{d x}{d s}, \\
V & =\frac{1}{2} \\
& \cdot \int_{0}^{L} \oint \sum_{k=1}^{N} \int_{h(k)} \rho^{(k)}\left\{\sigma_{z z}^{(k)}\left[w^{\prime}+y \theta_{x}^{\prime}-F_{w} \phi^{\prime \prime}-n\left(\frac{d x}{d s} \theta_{x}^{\prime}+a \phi^{\prime \prime}\right)\right]+\sigma_{s z}^{(k)}\left[u^{\prime} \frac{d x}{d s}+\left(v^{\prime}+\theta_{x}\right) \frac{d y}{d s}+\frac{2 A_{e}}{\beta} \phi^{\prime}\right]+\sigma_{n z}^{(k)}\left[\left(u^{\prime}\right) \frac{d y}{d s}-\left(v^{\prime}+\theta_{x}\right) \frac{d x}{d s}\right]\right\} d n d s d z,
\end{aligned}
$$

where $\sigma_{i j}^{k}$ denotes the stress component and represents the three-dimensional constitutive equations for the actuator layers with strain components $\varepsilon_{i j}$. It can be deduced as

$$
\begin{aligned}
{\left[\begin{array}{c}
\sigma_{s s} \\
\sigma_{z z} \\
\sigma_{s z}
\end{array}\right]=} & {\left[\begin{array}{ccc}
C_{11} & C_{12} & 0 \\
C_{12} & C_{11} & 0 \\
0 & 0 & \frac{C_{11}-C_{12}}{2}
\end{array}\right]\left[\begin{array}{c}
\varepsilon_{s s} \\
\varepsilon_{z z} \\
\varepsilon_{s z}
\end{array}\right] } \\
& -\left[\begin{array}{c}
e_{31} \xi_{3} R(n) R(s) R(z) \\
e_{31} \xi_{3} R(n) R(s) R(z) \\
0
\end{array}\right],
\end{aligned}
$$

where $C_{i j}$ means elastic coefficient and the last terms identify the actuation stresses induced by the applied electric field. In addition, $R$ is a spatial function expressed by Heaviside distribution [10]. The composite parameters and piezoelectric properties can be found in Table 1 .

Based on the extended Hamilton principle, the governing system of cantilever vibration can be derived as follows:

$$
\int_{t_{1}}^{t_{2}}(\delta T-\delta V+\delta W) d t=0
$$

where $\delta W$ is the virtual work of the nonconservative forces depicted as [10]

$$
\begin{aligned}
& \delta W=\int_{0}^{L}\left(F_{u} \delta u_{0}+F_{v} \delta v_{0}+M_{\varphi} \delta \phi-M_{x a} \delta \theta_{x}^{\prime}\right. \\
& \left.+B_{w a} \phi^{\prime \prime}\right) d z,
\end{aligned}
$$

where $M_{x a}$ and $B_{w a}$ are the piezoelectrically induced terms expressed as

$$
\begin{aligned}
M_{x a} & =\oint \xi_{3} b_{k}^{a} e_{31} R(s, z) \\
\cdot & {\left[y\left(1-\frac{A_{12}}{A_{11}}\right)+\frac{d x}{d s}\left(\frac{B_{12}}{A_{11}}-n_{k}^{a}\right)\right] d s, } \\
B_{w a} & =\oint \xi_{3} b_{k}^{a} e_{31} R(s, z) \\
. & {\left[F_{w}\left(1-\frac{A_{12}}{A_{11}}\right)-a\left(\frac{B_{12}}{A_{11}}-n_{k}^{a}\right)\right] d s, }
\end{aligned}
$$

where

$$
n_{k}^{a}=\frac{1}{2} c-b_{k}^{a}-\frac{1}{8} h, \quad a=-y(s) \frac{d y}{d s}-x(s) \frac{d x}{d s}
$$

and $\xi_{3}$ is the applied electric field on which the piezoelectrically induced moment depends related with patch area $A_{p}$. It is computed as

$$
\xi_{3} \xi_{3}^{p} A_{p}=\iint\left\{e_{31} y(s)\left[\dot{\theta}_{x}\left(z_{2}\right)-\dot{\theta}_{x}\left(z_{1}\right)\right]\right\} d s d t
$$

and $A_{i j}$ and $B_{i j}$ denote local stretching and stretching-bending coupling rigidity quantities, respectively.

It is complicated to directly deduce the equations of motion of the rotating section with piezoelectric fiber rein forced composite actuators. Hence another simplified method is applied here. Based on (6), the equations of motion of cantilever vibration can be obtained first. With the rotating centrifugal motion [11] and the CAS structural tailoring technology being considered [10], the equations of rotating 
motion with vertical bending-twist-transverse shear coupling can be deduced as

$$
\begin{aligned}
& b_{1} \ddot{v}-a_{1} v^{\prime \prime}-a_{2} \phi^{\prime \prime \prime}-a_{1} \theta_{x}^{\prime}-\frac{1}{2} m \Omega^{2}\left[v^{\prime \prime}\left(L^{2}-z^{2}\right)\right. \\
& \left.+v^{\prime}(-2 z)\right]=F \cos (\psi), \\
& {\left[b_{2} \ddot{\phi}-b_{3} \ddot{\phi}^{\prime \prime}+a_{3} \phi^{\prime \prime \prime \prime}-a_{5} \phi^{\prime \prime}+a_{2} v^{\prime \prime \prime}+a_{4} \theta_{x}^{\prime \prime}+B_{w a}^{\prime \prime}\right.} \\
& \left.\quad-\frac{1}{2} m \Omega^{2} K_{A}^{2}\left(L^{2}-z^{2}\right)\right] \phi^{\prime \prime}+m \Omega^{2} K_{A}^{2} z \phi^{\prime} \\
& \quad+m \Omega^{2}\left(K_{m 2}^{2}-K_{m 1}^{2}\right) \phi=M+F e \cos (\psi-\varphi), \\
& b_{0} \ddot{\theta}_{x}-a_{6} \theta_{x}^{\prime \prime}+\left(a_{2}-a_{0}\right) \phi^{\prime \prime}+a_{1} v^{\prime}+a_{1} \theta_{x}+M_{x a}^{\prime}=0,
\end{aligned}
$$

where the related composite structural parameters $a_{j}$ and $b_{j}$ are displayed in appendix, and the classical aerodynamics of lift $F$ and moment $M$ can be expressed as [2]

$$
\begin{gathered}
F=-\rho_{a} c V_{0}^{2} \pi\left(\alpha+v^{\prime}\right), \\
M=-\rho_{a} c^{2} V_{0}^{2} \pi e\left(\alpha+v^{\prime}\right),
\end{gathered}
$$

where

$$
\begin{aligned}
\Omega & =\frac{\lambda U}{L}, \\
K_{m 1}^{2} & =\frac{1}{m} \iint \rho_{m} x^{2} d y d x, \\
K_{m 2}^{2} & =\frac{1}{m} \iint \rho_{m} y^{2} d y d x, \\
K_{m}^{2} & =K_{m 1}^{2}+K_{m 2}^{2}, \\
A_{0} & =\iint d y d x, \\
A_{0} K_{A}^{2} & =\iint\left(y^{2}+x^{2}\right) d y d x .
\end{aligned}
$$

\section{Solution Methodology}

3.1. Application of Galerkin Method. To analyze the aeroelastic system given by (11a)-(11c), the Galerkin method is used so as to reduce the aeroelastic coupled equations into the statespace form [11]. Firstly the representation of displacement functions is as follows:

$$
\begin{gathered}
V(z, t)=V^{T}(z) q_{v}(t), \\
\phi(z, t)=\Phi^{T}(z) q_{\varphi}(t), \\
\theta_{x}(z, t)=S^{T}(z) q_{\theta}(t),
\end{gathered}
$$

where test functions are required to satisfy the kinematics and force boundary conditions of the cantilever blade and can be written as

$$
\begin{aligned}
V^{T}(z)= & {\left[v_{1}, v_{2}, v_{3}, v_{4}, \ldots, v_{N}\right], } \\
\Phi^{T}(z)= & {\left[\varphi_{1}, \varphi_{2}, \varphi_{3}, \varphi_{4}, \ldots, \varphi_{N}\right], } \\
S^{T}(z)= & {\left[\theta_{1}, \theta_{2}, \theta_{3}, \theta_{4}, \ldots, \theta_{N}\right], } \\
v_{j}(z)= & \cosh \left(\beta_{j} z\right)-\cos \left(\beta_{j} z\right) \\
& +\alpha_{j}\left(\sinh \left(\beta_{j} z\right)-\sin \left(\beta_{j} z\right)\right), \\
\varphi_{j}(z)= & \sqrt{2} \sin \left(\gamma_{j} z\right), \\
\theta_{j}(z)= & \sqrt{2} \sin \left(\gamma_{j} z\right), \\
\gamma_{j}= & \pi\left(j-\frac{1}{2}\right), \\
\beta_{j}= & \left(j-\frac{1}{2}\right) \pi, \\
\alpha_{j}= & -\frac{\cos \beta_{j}+\cosh \beta_{j}}{\sin \beta_{j}+\sinh \beta_{j}} .
\end{aligned}
$$

Secondly substituting (15) into (11a)-(11c), with Galerkin method applied, gives $3 N$ matrix equations as follows:

$$
M_{P N} \ddot{X}+K_{P N} X=Q_{P N},
$$

where the state variable $X=\left[\left.q_{v}\right|_{N \times 1} ^{T},\left.q_{\varphi}\right|_{N \times 1} ^{T},\left.q_{\theta}\right|_{N \times 1} ^{T}\right]^{T}$, and the related coefficient matrices are

$$
\begin{aligned}
& M_{P N}=\left[\begin{array}{lll}
M_{P N 1} & M_{P N 2} & M_{P N 3}
\end{array}\right] \text {, } \\
& M_{P N 1}=\left[\begin{array}{c}
\int_{0}^{L} b_{1} V^{T}(z) v_{j} d z \\
0 \\
0
\end{array}\right] \text {, } \\
& M_{P N 2}=\left[\int_{0}^{L}\left\{b_{2} \Phi^{T}(z)-b_{3}\left[\Phi^{T}(z)\right]^{\prime \prime}\right\} \varphi_{j} d z\right] \text {, }
\end{aligned}
$$$$
M_{P N 3}=\left[\begin{array}{c}
0 \\
0 \\
\int_{0}^{L} b_{0} S^{T}(z) \theta_{j} d z
\end{array}\right],
$$$$
K_{P N}=\left[\begin{array}{lll}
K_{P N 1} & K_{P N 2} & K_{P N 3}
\end{array}\right] \text {, }
$$ 


$$
\begin{aligned}
& K_{P N 1} \\
& =\left[\begin{array}{c}
\int\left(Q_{v}+\pi \rho_{a} c V_{0}^{2}\left[V^{T}(z)\right]^{\prime} \cos \psi\right) v_{j} d z \\
\int\left(a_{2}\left[V^{T}(z)\right]^{\prime \prime \prime}+\pi \rho_{a} V_{0}^{2}\left[V^{T}(z)\right]^{\prime} e c(c+\cos \psi)\right) \varphi_{j} d z \\
\int a_{1}\left[V^{T}(z)\right]^{\prime} \theta_{j} d z
\end{array}\right], \\
& K_{P N 2}=\left[\begin{array}{c}
-\int\left(a_{2}\left[\Phi^{T}(z)\right]^{\prime \prime \prime}+\pi \rho_{a} c V_{0}^{2}\left[\Phi^{T}(z)\right] \cos \psi\right) v_{j} d z \\
\int\left(Q_{\varphi}-\pi \rho_{a} V_{0}^{2}\left[\Phi^{T}(z)\right] e c(c+\cos \psi)\right) \varphi_{j} d z \\
\int\left(a_{2}-a_{0}\right)\left[\Phi^{T}(z)\right]^{\prime \prime} \theta_{j} d z
\end{array}\right], \\
& K_{P N 3}=\left[\begin{array}{c}
-\int a_{1}\left[S^{T}(z)\right]^{\prime} v_{j} d z \\
\int\left\{\left.P_{B w a}\left[S^{T}(z)\right]^{\prime \prime}\right|_{z=L}+a_{4}\left[S^{T}(z)\right]^{\prime \prime}\right\} \varphi_{j} d z \\
\int Q_{\theta} \theta_{j} d z
\end{array}\right], \\
& Q_{P N}=\left[\begin{array}{c}
-\int\left(\pi \rho_{a} c V_{0}^{2} \psi \cos \psi\right) v_{j} d z \\
-\int\left(\pi \rho_{a} V_{0}^{2} \psi e c(c+\cos \psi)\right) \varphi_{j} d z \\
0
\end{array}\right],
\end{aligned}
$$

where

$$
\begin{aligned}
& Q_{v}=-a_{1}\left[V^{T}(z)\right]^{\prime \prime}-\left(\frac{m \Omega^{2}}{2}\right)\left(L^{2}-z^{2}\right)\left[V^{T}(z)\right]^{\prime \prime} \\
& +m \Omega^{2} z\left[V^{T}(z)\right]^{\prime} \\
& Q_{\varphi}=a_{3}\left[\Phi^{T}(z)\right]^{\prime \prime \prime \prime}-a_{5}\left[\Phi^{T}(z)\right]^{\prime \prime} \\
& +m \Omega^{2} k_{A}^{2} z\left[\Phi^{T}(z)\right]^{\prime}+m \Omega^{2}\left(k_{m 2}^{2}-k_{m 1}^{2}\right) \\
& \cdot z\left[\Phi^{T}(z)\right]-\left(\frac{m \Omega^{2}}{2}\right) k_{A}^{2}\left(L^{2}-z^{2}\right)\left[\Phi^{T}(z)\right]^{\prime \prime}, \\
& Q_{\theta}=-a_{6}\left[S^{T}(z)\right]^{\prime \prime}+a_{1}\left[S^{T}(z)\right]+\left.\left[S^{T}(z)\right]^{\prime}\right|_{z=L} \\
& \left\{\frac{4 e_{31} b_{k}^{a}}{t_{a}}\left(\frac{\xi_{3} t_{a}}{\left[S^{T}(L) q_{\theta}\right]}\right)\right. \\
& \left.\oint \oint\left[y\left(1-\frac{A_{12}}{A_{11}}\right)+\frac{d x}{d s} \frac{B_{12}}{A_{11}}-\frac{d y}{d s} n_{k}^{a}\right] d s\right\}, \\
& P_{B w a}=\frac{4 e_{31} b_{k}^{a}}{t_{a}}\left(\frac{\xi_{3} t_{a}}{\left[S^{T}(L) q_{\theta}\right]}\right) \\
& \left.\oint \oint F_{w}\left(1-\frac{A_{12}}{A_{11}}\right)-a \frac{B_{12}}{A_{11}}+a n_{k}^{a}\right] d s .
\end{aligned}
$$

Equation (17) is the linear decoupling equation governing the motion of the aeroelastic system, which is a matrix equation with $3 N$ subequation structure.

3.2. Active Control. In active flutter suppression, it is essential to properly select actuators and properly use control method and mathematical algorithm. The feedback control is achieved through the piezoelectrically induced vertical transverse shear motion at the blade tip, considered in conjunction with the implementation of a combined feedback control law, when external voltage of opposite sign is applied in the upper and bottom piezoactuator layer. The applied electric field $\xi_{3}$ on which the piezoelectrically induced moment depends may be expressed through a prescribed linear functional relationship with the kinematical response quantities characterizing the blade's response. The piezoelectrically induced bending moment $M_{x a}$ intervenes solely in the boundary conditions prescribed at the blade tip and plays the role of a boundary moment control due to special distribution of piezoactuators [12]. A feedback control law is implemented with $M_{x a}$ at the blade tip expressed as

$$
M_{x a}(L, t)=k_{v} \dot{\theta}_{x}(L, t)+k_{a} \ddot{\theta}_{x}(L, t),
$$

where $k_{v}$ and $k_{a}$ denote the velocity and acceleration feedback gains, respectively. So for active control with feedback law, system equation (17) is rewritten as

$$
M_{P N} \ddot{X}+C_{P N} \dot{X}+K_{P N} X=Q_{P N},
$$

where the new coefficient matrices are as follows:

$$
\begin{aligned}
& M_{P N 3}=\left[\begin{array}{c}
0 \\
0 \\
\int_{0}^{L}\left\{b_{0} S^{T}(z)+\left.K_{a}\left[S^{T}(z)\right]^{\prime}\right|_{z=L}\right\} \theta_{j} d z
\end{array}\right], \\
& C_{P N}=\left[\begin{array}{ccc}
0 & 0 & 0 \\
0 & 0 & 0 \\
0 & 0 & \left.\int K_{v}\left[S^{T}(z)\right]^{\prime}\right|_{z=L} \theta_{j} d z
\end{array}\right] .
\end{aligned}
$$

In order to determine the time domain response of the dynamic system of (21), a Runge-Kutta time-marching approach is applied. Using the method presented in [1], (21) will be expressed in state-space form. In general, upon defining the state vector $Y=\left[X^{T}, \dot{X}^{T}\right]^{T}$ and adjoining the identity equation $\dot{X}=\dot{X},(21)$ can be converted to the statespace expression:

$$
\begin{aligned}
\dot{Y} & =A Y+B, \\
Y_{O} & =C Y+D,
\end{aligned}
$$

where $6 N \times 6 N$ state matrix $A$ and $6 N \times 1$ matrix $B$ are given by

$$
\begin{aligned}
& A=\left[\begin{array}{cc}
0 & I_{E} \\
-M_{P N}^{-1} K_{P N} & -M_{P N}^{-1} C_{P N}
\end{array}\right], \\
& B=\left[\begin{array}{c}
0 \\
M_{P N}^{-1} Q_{P N}
\end{array}\right] .
\end{aligned}
$$

Herein $I_{E}$ is the $3 N$ unitary matrix, $C$ is $6 N$ unitary matrix, and $D$ is $6 N \times 1$ zero matrix. 
In order to minimize settling time and required control energy, optimal control techniques are necessary for controller design. Since these sensitive structures often experience time-varying loads, their safe and effective design requires accurate response [13]. The linear quadratic regulator (LQR) is often used to analyze vibration control of rotating composite beam. An advantage of the quadratic optimal control method is that it provides a systematic way of computing the state feedback control gain matrix [14]. In [13], the Galerkin method, along with either instantaneous or classical LQR methods, is used to analyze vibration control of a rotating composite pretwisted single-celled box beam, exhibiting transverse shear flexibility and restrained warping.

Here in order to suppress the too large initial vibration amplitude in LQR process and decrease the influence of measurement noise (might be produced by unsteady aerodynamics), the linear quadratic Gaussian (LQG) controller is used $[4,15]$. It is assumed that the measurement noise and disturbance signals (process noise) are stochastic with known statistical properties and are hidden in system equation (23). The dynamic equation of LQG controller can be represented as

$$
\begin{aligned}
& \dot{X}_{C}=A_{C} X_{C}+B_{C} u, \\
& Y_{C}=C_{C} X_{C}
\end{aligned}
$$

where

$$
\begin{aligned}
& A_{C}=A-B K_{R}-K_{F} C+K_{F} D K_{R}, \\
& B_{C}=K_{F}=X_{F} C^{T} R_{1}^{-1}, \\
& C_{C}=-K_{R}=-\left(R+D^{T} Q D\right)^{-1}\left(B^{T} X_{R}+D^{T} Q C\right),
\end{aligned}
$$

where $Q$ and $R$ are the weighting matrices of system outputs and control inputs, respectively; $K_{F}$ and $K_{R}$ are the gain matrices of the Kalman state estimator and the gain matrix of the optimal regulator, respectively; $X_{F}$ and $X_{R}$ are the positive finite solution of algebraic Riccati equations as follows:

$$
\begin{aligned}
& A X_{F}+X_{F} A^{T}-X_{F} C^{T} R_{1}^{-1} C X_{F}+Q_{1}=0, \\
& A^{T} X_{R}+X_{R} A-\left(C^{T} Q D+X_{R} B\right)\left(R+D^{T} Q D\right)^{-1} \\
& \quad \cdot\left(B^{T} X_{R}+D^{T} Q C\right)+C^{T} Q C=0,
\end{aligned}
$$

where $Q_{1}$ and $R_{1}$ are the intensity matrices of the gust input and the Gaussian white noise process of measurement, respectively. For the LQG control law, the gain matrix $K_{R}$ can be determined firstly by choosing the weighting matrices as $Q$ being $6 N$ unitary matrix and $R=0.001$. Then the gain matrix $K_{F}$ of the Kalman state estimator is determined via $Q_{1}=1$ and $R_{1}$ being $6 N$ unitary matrix.

The LQG control problem is to find the optimal control $u(t)$ which minimizes

$$
J=E\left\{\lim _{T \rightarrow \infty} \frac{1}{T} \int_{0}^{T}\left[Y^{T} Q Y+u^{T} R u\right] d t\right\}
$$

and the required solution to the LQG problem is then found by replacing $Y$ by $X_{C}$, to give the expression

$$
u(t)=-K_{R} X_{C}(t)
$$

\section{Results and Discussion}

To testify the validity of active flutter suppression, numerical results of time responses at blade tip for LQG controllers are presented, compared with related results of other cases. Generally, rotating blades have specific rotating speed, ply angle, and tip speed ratio for operation. Here basic testing parameters are ply angle $\theta_{p}=30^{\circ}$; tip speed ratio of wind turbine $\lambda=3$; blade length $L=1.5 \mathrm{~m}$.

In general, the critical wind speed of a $2 \mathrm{D}$ section can be determined by $\mathrm{v}-\mathrm{g}$ method [4] by eigenvalue analysis. However, the object of present research is a $3 \mathrm{D}$ entity with bending-twist-transverse shear coupling. After decoupling the number of subequations in (17) reaches $3 N$ (here $N \geq 5$ ), with the number of eigenvalues being $6 \mathrm{~N}$. V-g method has lost the utility. However, we can use another approximate method by the eigenvalue analysis. For different wind speeds, the maximum value of all the eigenvalues of each wind speed is determined, and then the critical wind speed is determined from the fluctuation of the largest eigenvalues. Figure 2(a) displays maximum real parts of eigenvalues versus velocities from $20 \sim 45 \mathrm{~m} / \mathrm{s}$. It can be obviously demonstrated that the critical wind speed is $U=34.69 \mathrm{~m} / \mathrm{s}$.

For flutter analysis, an approximate critical state range based on $U=34.69 \mathrm{~m} / \mathrm{s} \sim 34.695 \mathrm{~m} / \mathrm{s}$ is considered. When $U<$ $34.69 \mathrm{~m} / \mathrm{s}$, the system is convergent with smaller amplitude. When $U>34.695 \mathrm{~m} / \mathrm{s}$, the system presents the divergent state with a large amplitude.

In general, the damping ratio increases constantly as the feedback control gain increases. However feedback control gain cannot be increased infinitely because the applied voltage must be limited for the sake of breakdown voltage of actuators [16]. According to the requirements of blade structure and actual control hardware here, the fixed feedback gains of $k_{v}=1$ and $k_{a}=1$ are applied.

Figure 2(b) shows the time responses of vertical bending $(v)$, twist $(\phi)$, and transverse shear $\left(\theta_{x}\right)$ motions under situation of without piezoelectric materials. Two critical wind speed values are considered at the same time; one is $U=$ $34.69 \mathrm{~m} / \mathrm{s}$ (in blue mark) and the other one is $U=34.695 \mathrm{~m} / \mathrm{s}$ (in green mark).

From the whole response trend in the case of $U=$ $34.69 \mathrm{~m} / \mathrm{s}$, the three displacements seem to be convergent. In fact for the vertical bending displacement $v$, within $2 \mathrm{~s}$ time, the amplitude of the vibration quickly exceeds the length of the blade $L$, so actually the vertical bending $(v)$ motion has been in the state of divergence, so do twist $(\phi)$ and transverse shear $\left(\theta_{x}\right)$ motions. The instability also can be testified by Imag.-Real plot of eigenvalues of homogeneous equation system in Figure 3(a), where some closed-loop poles lie in the right-half plane, resulting in an unstable system.

As for another case of $U=34.695 \mathrm{~m} / \mathrm{s}$ in Figure 2(b), the three displacements rapidly diverge from the start with 

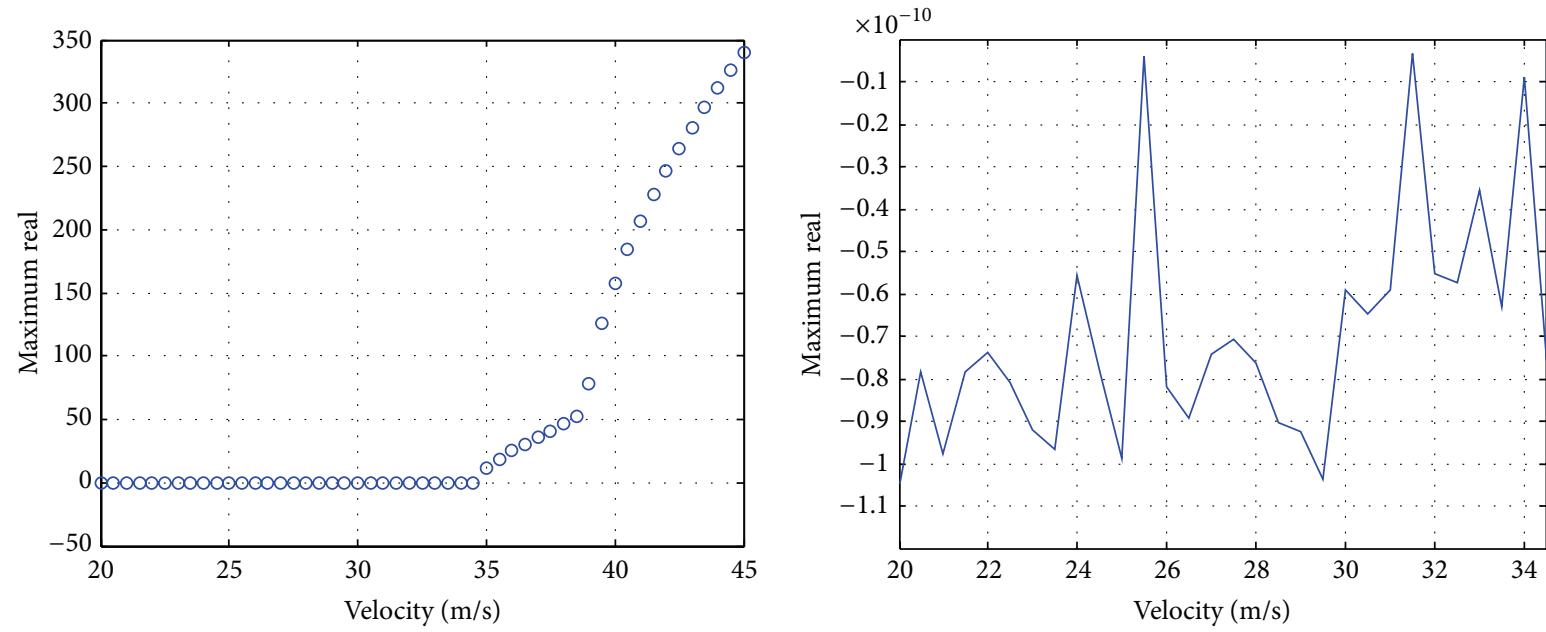

(a) Maximum real parts of eigenvalues versus velocities
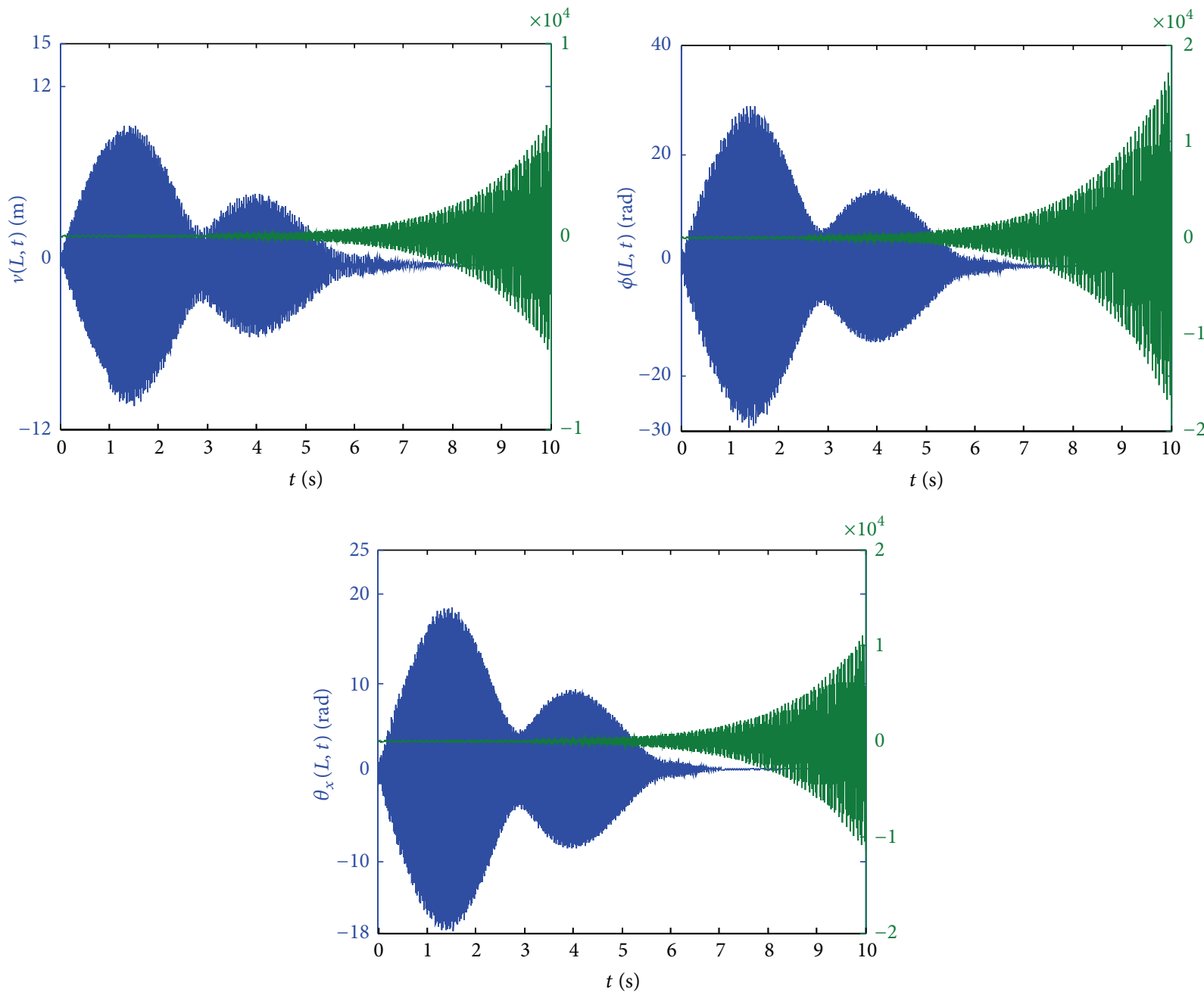

$U=30.69 \mathrm{~m} / \mathrm{s}$

$U=30.695 \mathrm{~m} / \mathrm{s}$

(b) The responses of vertical bending $(v)$, twist $(\phi)$, and transverse shear $\left(\theta_{x}\right)$ motions for both $U=34.69 \mathrm{~m} / \mathrm{s}$ and $U=34.695 \mathrm{~m} / \mathrm{s}$

FIGURE 2: Maximum real parts of eigenvalues versus velocities (a) and the responses for both $U=34.69 \mathrm{~m} / \mathrm{s}$ and $U=34.695 \mathrm{~m} / \mathrm{s}$ under situation of without piezoelectric materials (b). 


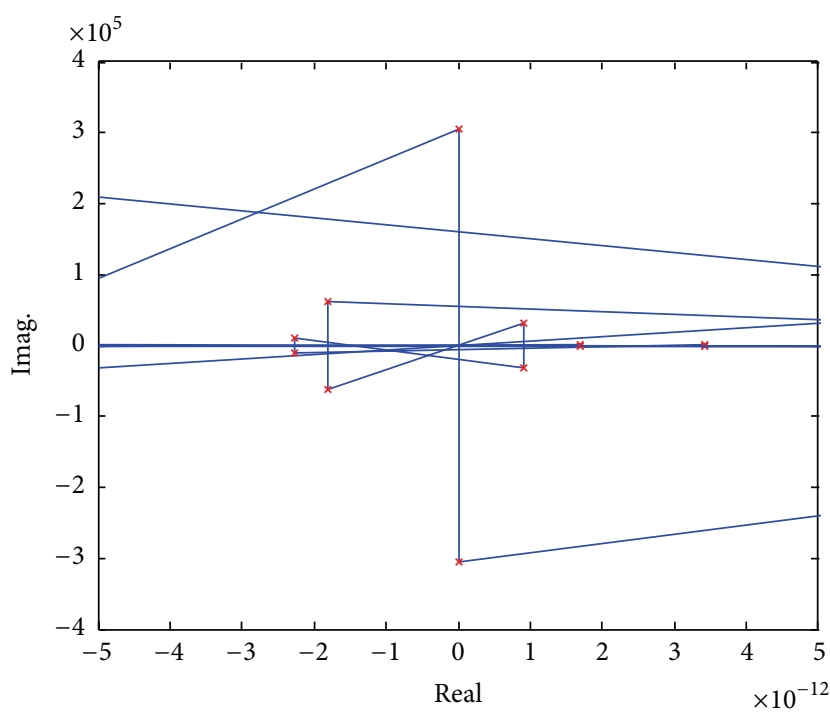

(a) Without piezoelectric materials

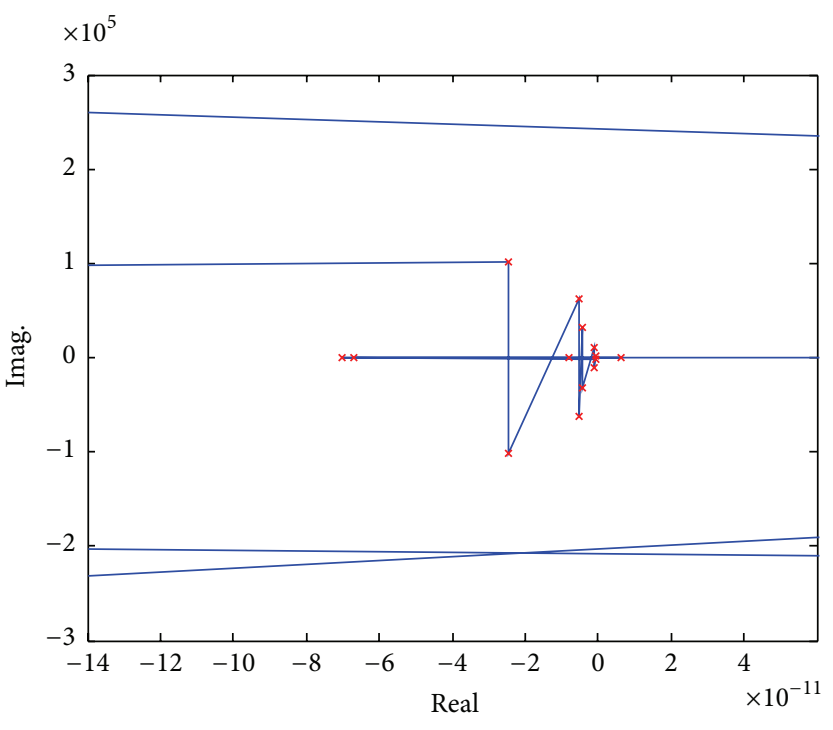

(b) With gains of $k_{v}=1$ and $k_{a}=1$, but without optimal controller

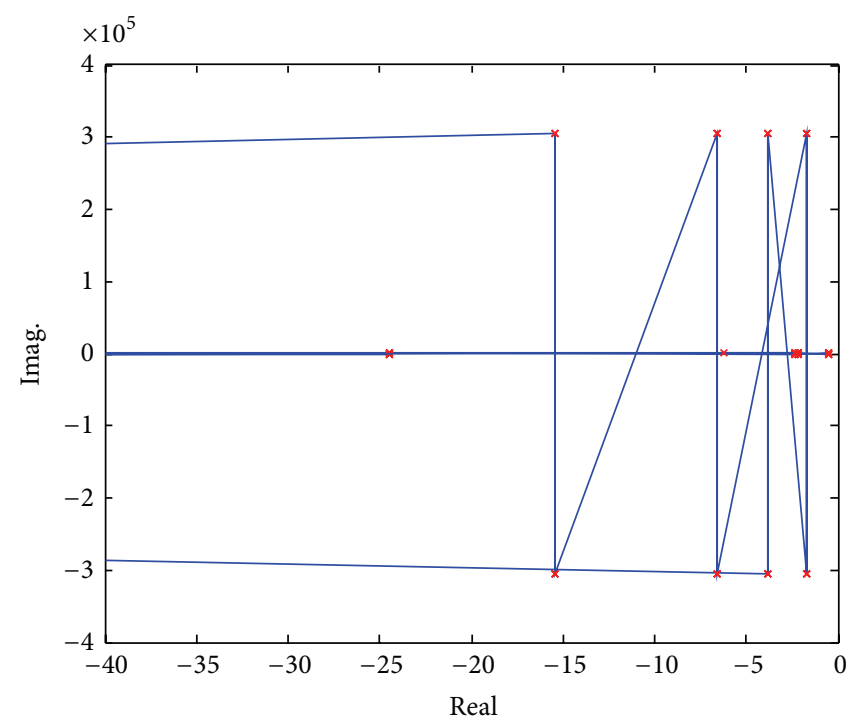

(c) With LQG controller

FIGURE 3: Imag.-Real plot of homogeneous equation system for $U=34.69 \mathrm{~m} / \mathrm{s}$ defined in state-space.

larger amplitude. These two cases in Figure 2(b) both can be regarded as the unstable states.

Take the case of $U=34.69 \mathrm{~m} / \mathrm{s}$; for example, analysis and discussion for active control and flutter suppression will be carried out in the following in Figures 3 5.

Figure 3 shows Imag.-Real plot of homogeneous equation system (only concerning the characteristic matrices of $A$ and $A_{C}$ ) for $U=34.69 \mathrm{~m} / \mathrm{s}$ defined in state-space: (a) without piezoelectric materials; (b) with gains of $k_{v}=1$ and $k_{a}=1$, but without optimal controller; (c) with LQG controller. Figures 3(a) 3(b) show the system instability. Some closed-loop poles lie in the right-half plane, and others are in the left-half plane. It can be seen from the Imag.-Real plot of Figure 3(c) that the homogeneous equation system obtained by control of LQG controller is stable, with all the closed-loop poles being in the left-half plane.

Figure 4 shows comparisons of the three displacements responses for $U=34.69 \mathrm{~m} / \mathrm{s}$ with fixed feedback gains of $k_{v}=$ 1 and $k_{a}=1$, which are characterized by the state without optimal controller (in blue mark) and LQG controller (in red mark).

It can be seen that piezoelectric actuation based on fixed feedback gains of $k_{v}=1$ and $k_{a}=1$ and without optimal controller (blue mark in Figure 4) can greatly suppress the flutter, compared with the results in Figure 2(b). However, the results of these three displacements still maintain a larger vibration amplitude, and what is more, combined with the eigenvalue analysis in Figure 3(b), the system (with blue mark 


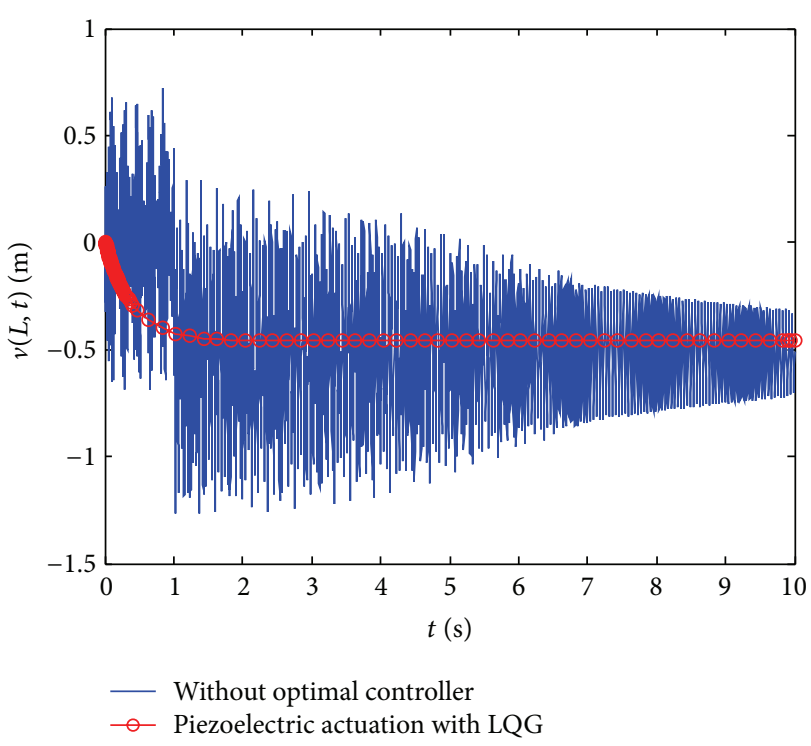

(a)

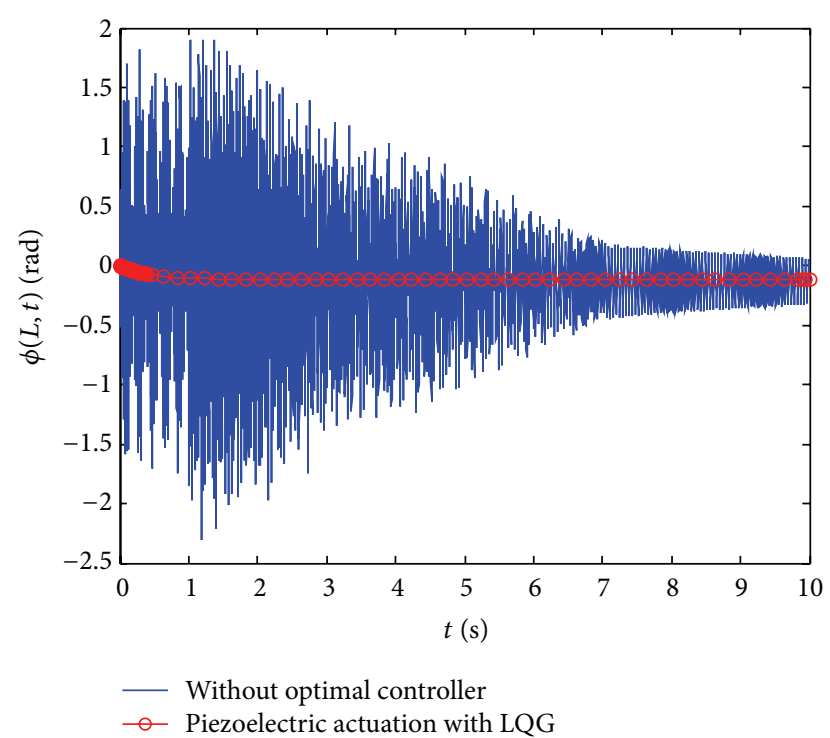

(b)

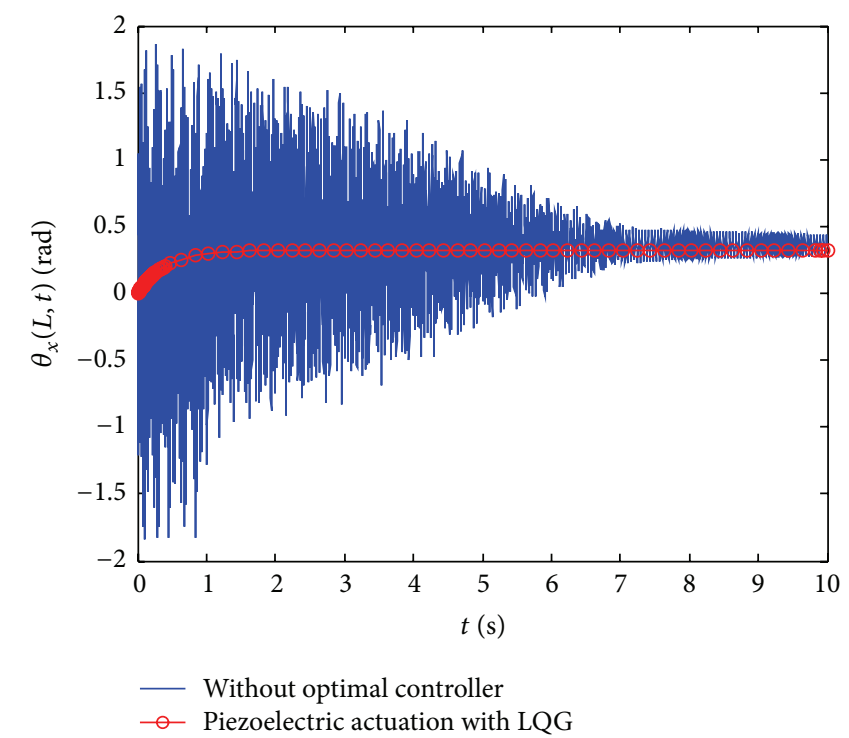

(c)

FIGURE 4: The responses of the three displacements under condition of fixed feedback gains of $k_{v}=1$ and $k_{a}=1$, which are characterized by the state without optimal controller (in blue mark) and LQG controller (in red mark) for $U=34.69 \mathrm{~m} / \mathrm{s}$.

in Figure 4) might be conditionally stable. In practice, conditionally stable systems are not desirable. If system parameter assumes a value corresponding to unstable operation, the system may break down or may become nonlinear due to a saturation nonlinearity that may exist. Therefore, further active control based on theoretical algorithm, such as LQG algorithm, is necessary.

As far as the trend of response is concerned, the three displacements based on LQG controller in Figure 4 are rapidly convergent from the start. Figure 4 (red mark) also shows that the flutter amplitudes of all the three displacements decrease rapidly with the change of time and tend to be steady within
2 s. It shows obvious effect of LQG flutter suppression on aeroelastic instability.

In contradistinction with the trends without feedback control occurring in Figure 2(b) for $U=34.69 \mathrm{~m} / \mathrm{s}$, the results displayed in Figure 4 reveal that LQG algorithm and active feedback control law have positive effects on classical flutter suppression. In order to verify the LQG law of universal, a further validation of LQG controller concerns the case of rapidly divergent state characterized by wind velocity of $U=38 \mathrm{~m} / \mathrm{s}$ without piezoelectric materials in Figure 5 (blue mark). From this figure it becomes apparent that when the wind speed is greater than the critical value, with increase of 


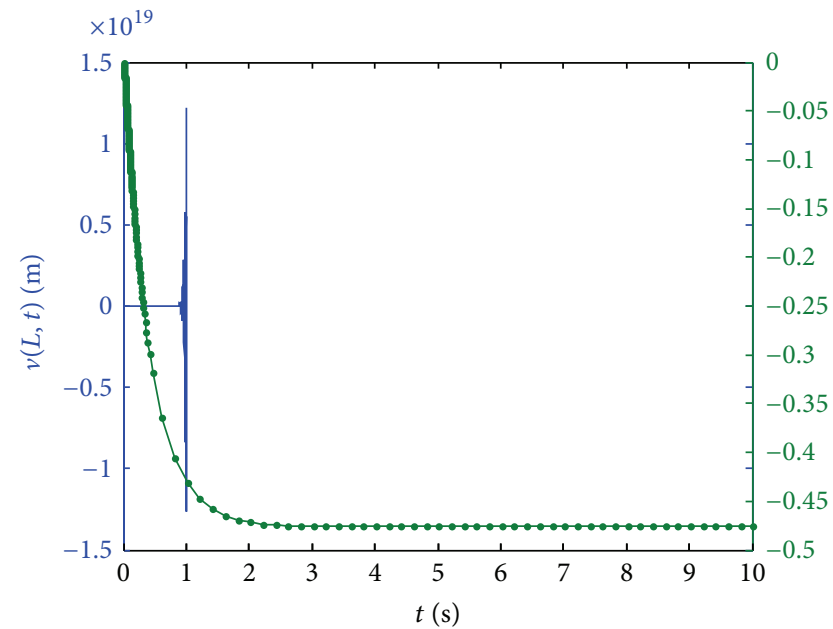

— Without piezoelectric materials
$\ldots$ Piezoelectric actuation with LQG

(a)

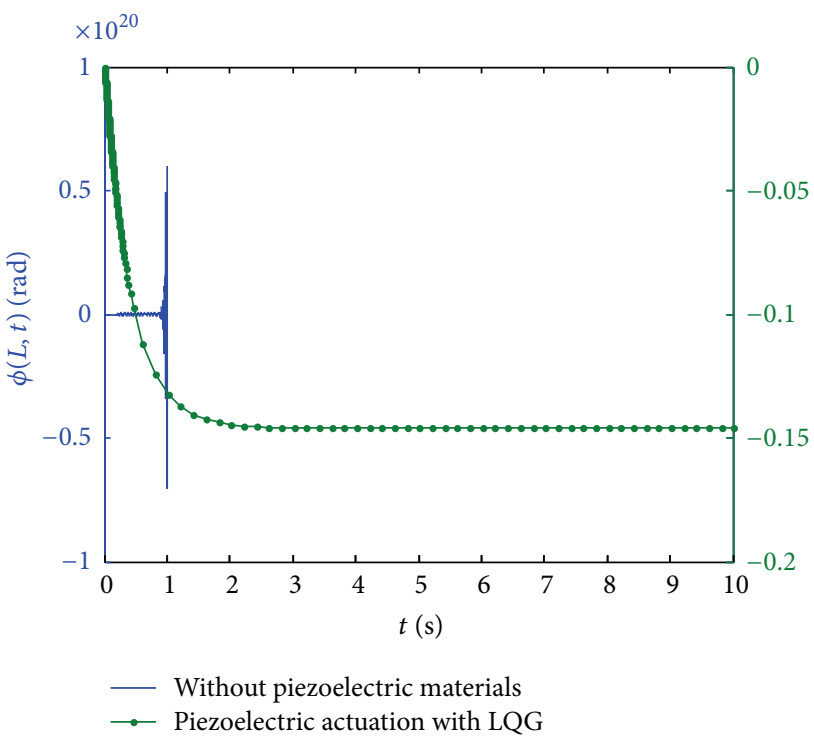

(b)

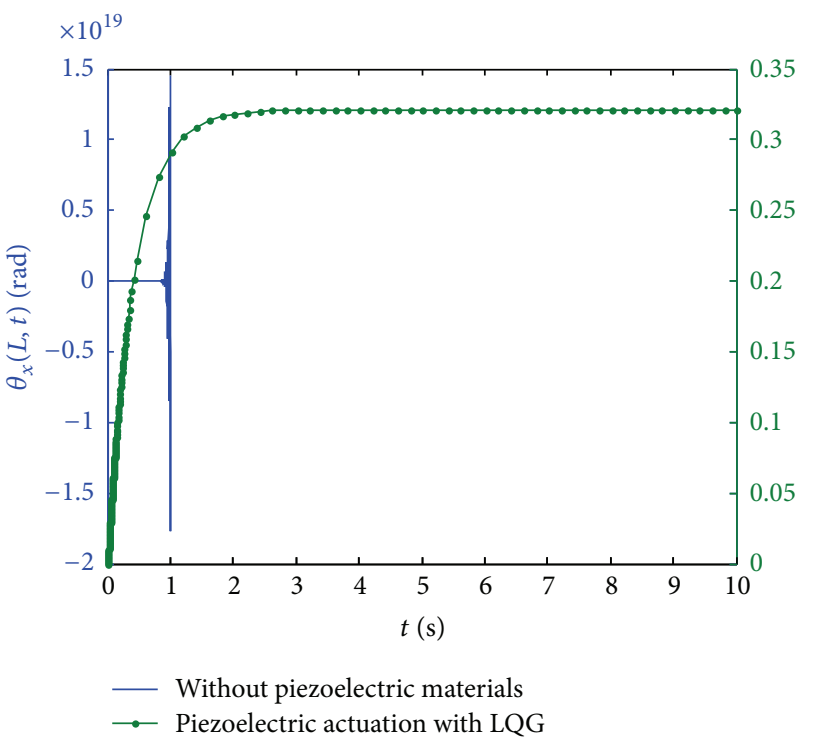

(c)

Figure 5: The time responses of the three displacements for wind velocity of $U=38 \mathrm{~m} / \mathrm{s}$, which are characterized by the state without piezoelectric materials and actuation by LQG controller.

time (within $1 \mathrm{~s}$ ), the magnitudes of the vibration amplitudes of the three displacements increase rapidly, even to unbelievable extent.

The effect of LQG controller on flutter suppression is illustrated in Figure 5 (green mark). The results show an expected conclusion, namely, that, for wind speed beyond the critical value, even in the adverse circumstances, the effect of LQG controller on flutter suppression is prominent. As a matter of fact, all the three responses under LQG controller are always stabilized to a constant value with smaller deviation when the time is extended beyond $2 \mathrm{~s}$; meantime the three velocities at the blade tip are stabilized to zero within $2 \mathrm{~s}$ (see Figure 6), which verify the control performance for the state variables of (29).
It should be stated that the purpose of present study is to investigate the validity of piezoelectric actuation under extreme conditions for $U \geqslant 34.69 \mathrm{~m} / \mathrm{s}$ as mentioned in Figures 2(b) 6. Of course, in general, the LQG algorithm is effective. A further validation of LQG controller concerns that the cases of low velocity for $U=10 \mathrm{~m} / \mathrm{s}$ and $U=$ $5 \mathrm{~m} / \mathrm{s}$ are displayed in Figure 7. The results show the same conclusion as Figure 5, namely, that, for wind speed in low area, the effect of LQG controller on flutter suppression is obvious. Simultaneously all the three responses under LQG controller are always stabilized to constant values when the time is extended beyond $3 \mathrm{~s}$.

In addition, the cost of piezoelectric materials is high with the mass of the system increasing as more actuator is 


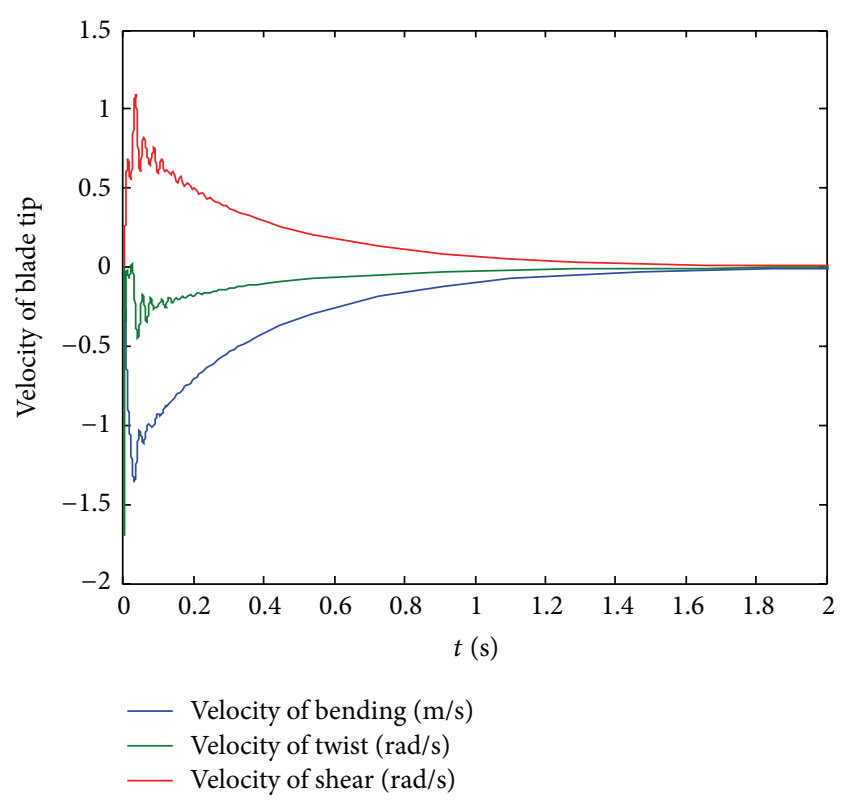

Figure 6: The three velocities at the blade tip.

used, with greater length and thickness being applied. Hence, adequate flutter suppression performance with LQG controller might be obtained through the proper arrangement and distributed size of sensor/actuator pair.

\section{Conclusions}

The purpose of present research is to investigate the validity of piezoelectric actuation under extreme conditions. An analytical study devoted to the mathematical modeling of single-cell thin-walled composite wind turbine blade beam is presented in the paper. Simultaneously classical flutter and active control based on piezoelectric actuation are investigated. The validity of the piezoelectric actuation is tested and illustrated by time domain response analysis. Since the related research on wind turbine is scarce, only numerical simulation technology is investigated here. The numerical illustrations reveal validation of the solution methodology and control algorithm used in the paper. Some concluding remarks can be drawn from the results as follows:

(1) Flutter suppression for classical flutter blade with vertical bending-twist-transverse shear coupling is investigated and discussed. Galerkin method is used to solve the coupling aeroelastic equations.

(2) Piezoelectric actuation is realized by active controller with feedback gains. It is obviously demonstrated that the LQG controller is robust and the effect on flutter suppression is apparent.

(3) It should be stated that the performance of LQG regulation depends on the choice of weighting matrices $Q, R$, and $Q_{1}, R_{1}$ which have no analytic solution. Hence in some sense, the LQG controller in fact is man-made.

\section{Appendix}

\section{Structural Parameters and Rigidity Quantities}

Consider

$$
\begin{aligned}
& a_{1}= \oint\left(K_{22} \frac{d y}{d s} \frac{d y}{d s}+K_{44} \frac{d x}{d s} \frac{d x}{d s}\right) d s, \\
& a_{2}=-\oint\left(F_{w} K_{12} \frac{d y}{d s}+K_{24} a \frac{d y}{d s}\right) d s, \\
& a_{3}=-\oint\left(F_{w}^{2} K_{11}+2 K_{14} F_{w} a+K_{44} a^{2}\right) d s, \\
& a_{4} \\
&=-\oint\left(F_{w} K_{12} \frac{d y}{d s}+K_{24} a \frac{d y}{d s}\right) d s \\
& K_{22}=A_{66}-\frac{A_{16}^{2}}{A_{11}}, \\
& a_{5}= \oint\left(2 \frac{A_{e}}{\beta} K_{23}\right) d s, \\
&\left.K_{13}-K_{43} \frac{d x}{d s}\right) d s, B_{22}-\frac{A_{12} B_{12}}{A_{11}}, \\
& a_{6}= \oint\left(K_{11} y^{2}-2 y K_{14} \frac{d x}{d s}+K_{44} \frac{A_{e}}{d s} \frac{d x}{d s}\right) d s, \\
& K_{12}= A_{26}-\frac{A_{12} A_{16}}{A_{11}}, \\
&\left(b_{1}, b_{4}, b_{5}, b_{10}\right)=\oint m_{0}\left(1, y^{2}, x^{2}, F_{w}^{2}\right) d s, \\
&\left(b_{14}, b_{15}, b_{18}\right)=\oint m_{2}\left[\left(\frac{d x}{d s}\right)^{2},\left(\frac{d y}{d s}\right)^{2}, a^{2}\right] d s, \\
& b_{0}=b_{4}+b_{5}, \\
& b_{2}=b_{4}+b_{5}, \\
& b_{3}=b_{10}+b_{18}, \quad \rho(k)\left(1, n^{2}\right) d n, \\
& K_{h(k-1)}, \frac{A_{12}^{2}}{A_{11}},
\end{aligned}
$$



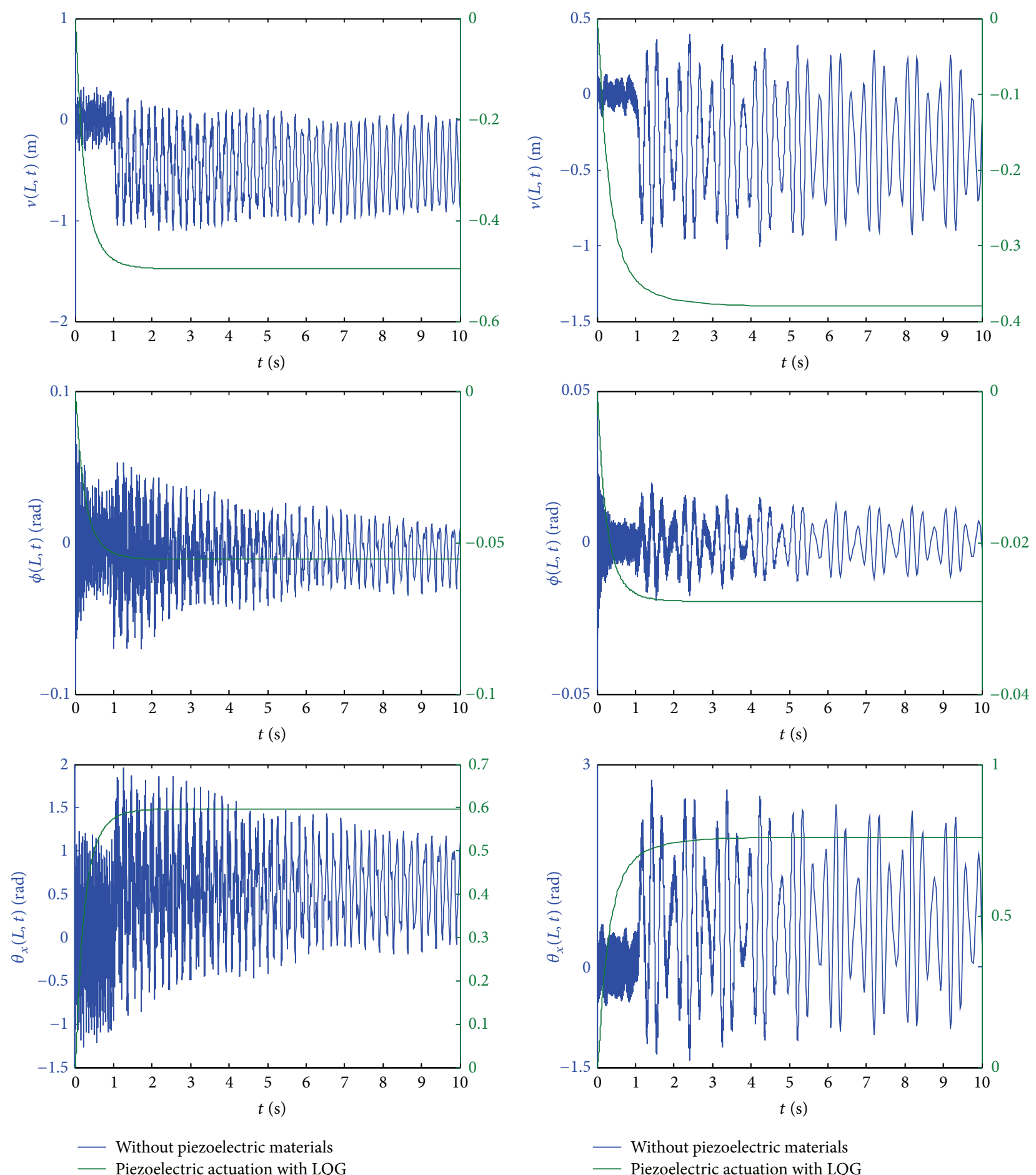

Figure 7: The time responses of the three displacements for wind velocities of $U=10 \mathrm{~m} / \mathrm{s}(\mathrm{a})$ and $U=5 \mathrm{~m} / \mathrm{s}$ (b), which are characterized by the state without piezoelectric materials and actuation by LQG controller.

$$
\begin{aligned}
& K_{23}=2 K_{22} \frac{A_{e}}{\beta}, \\
& K_{24}=B_{26}-\frac{A_{16} B_{12}}{A_{11}}, \\
& K_{43}=2 K_{24} \frac{A_{e}}{\beta},
\end{aligned}
$$

$$
\begin{aligned}
& K_{44}=D_{22}-\frac{B_{12}^{2}}{A_{11}}, \\
& \left(A_{i j} ; B_{i j} ; D_{i j}\right)=\sum_{k=1}^{N} \int_{h(k-1)}^{h(k)}\left(\bar{Q}_{i j}\right)_{k}\left(1 ; n ; n^{2}\right) d n .
\end{aligned}
$$




\section{Conflict of Interests}

The author declares that there is no conflict of interests regarding the publication of this paper.

\section{Acknowledgment}

This work is supported by the Natural Science Foundation of Shandong Province of China (Grant ZR2013AM016).

\section{References}

[1] T. Liu, "Aeroservoelastic pitch control of stall-induced flap/lag flutter of wind turbine blade section," Shock and Vibration, vol. 2015, Article ID 692567, 20 pages, 2015.

[2] T. Liu, "Flutter and response of composite wind turbine blade with bending-torsional coupling," Journal of Chemical and Pharmaceutical Research, vol. 6, no. 5, pp. 1544-1550, 2014.

[3] R. Drazumeric, B. Gjerek, F. Kosel, and P. Marzocca, "On bimodal flutter behavior of a flexible airfoil," Journal of Fluids and Structures, vol. 45, pp. 164-179, 2014.

[4] W. Qian, R. Huang, H. Hu, and Z. Yonghui, "Active flutter suppression of a multiple-actuated-wing wind tunnel model," Chinese Journal of Aeronautics, vol. 27, no. 6, pp. 1451-1460, 2014.

[5] H. Sun, L. Yang, and H. Li, "Sensitive flutter parameters analysis with respect to flutter-free design of compressor blade," Procedia Engineering, vol. 99, pp. 1597-1603, 2015.

[6] S. Kapuria and M. Y. Yasin, "Active vibration suppression of multilayered plates integrated with piezoelectric fiber reinforced composites using an efficient finite element model," Journal of Sound and Vibration, vol. 329, no. 16, pp. 3247-3265, 2010.

[7] P. Phung-Van, L. De Lorenzis, C. H. Thai, M. Abdel-Wahab, and H. Nguyen-Xuan, "Analysis of laminated composite plates integrated with piezoelectric sensors and actuators using higherorder shear deformation theory and isogeometric finite elements," Computational Materials Science, vol. 96, pp. 495-505, 2015.

[8] S. M. Hasheminejad and M. A. Motaaleghi, "Aeroelastic analysis and active flutter suppression of an electro-rheological sandwich cylindrical panel under yawed supersonic flow," Aerospace Science and Technology, vol. 11, no. 2-3, pp. 136-145, 2007.

[9] J.-S. Park and J.-H. Kim, "Design and aeroelastic analysis of active twist rotor blades incorporating single crystal macro fiber composite actuators," Composites Part B, vol. 39, no. 6, pp. 10111025,2008

[10] N. Sungsoo, Control of dynamic response of thin-walled composite beams using structural tailoring and piezoelectric actuation [Ph.D. thesis], Virginia Polytechnic Institute and State University, 1997.

[11] T. Liu, Y. Ren, and X. Yang, "Nonlinear aeroelastic stability analysis of wind turbine blade with bending-bending-twist coupling," Journal of Fluids and Structures, vol. 42, pp. 488-502, 2013.

[12] L. Librescu and S. Na, "Active vibration control of doubly tapered thin-walled beams using piezoelectric actuation," ThinWalled Structures, vol. 39, no. 1, pp. 65-82, 2001.

[13] C. D. Shete, N. K. Chandiramani, and L. Librescu, "Optimal control of a pretwisted shearable smart composite rotating beam," Acta Mechanica, vol. 191, no. 1-2, pp. 37-58, 2007.

[14] O. Katsuhiko, Modern Control Engineering, Prentice Hall, Upper Saddle River, NJ, USA, 5th edition, 2009.
[15] S. Skogestad and I. Postlethwaite, Multivariable Feedback Control: Analysis and Design, Wiley, Chichester, UK, 2nd edition, 2005.

[16] S.-C. Choi, J.-S. Park, and J.-H. Kim, "Active damping of rotating composite thin-walled beams using MFC actuators and PVDF sensors," Composite Structures, vol. 76, no. 4, pp. 362-374, 2006. 

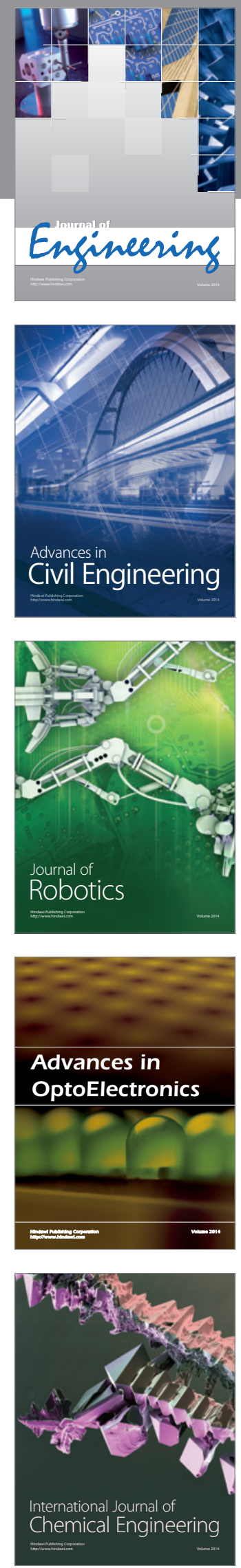

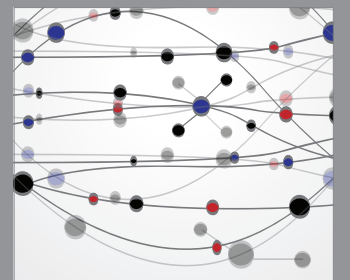

The Scientific World Journal
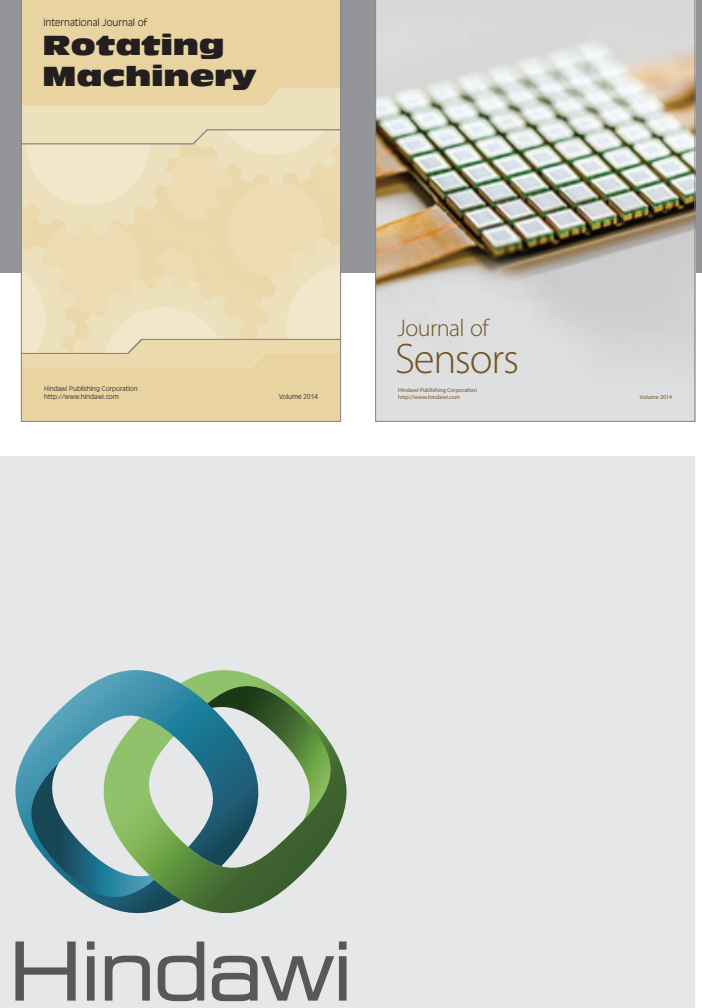

Submit your manuscripts at http://www.hindawi.com
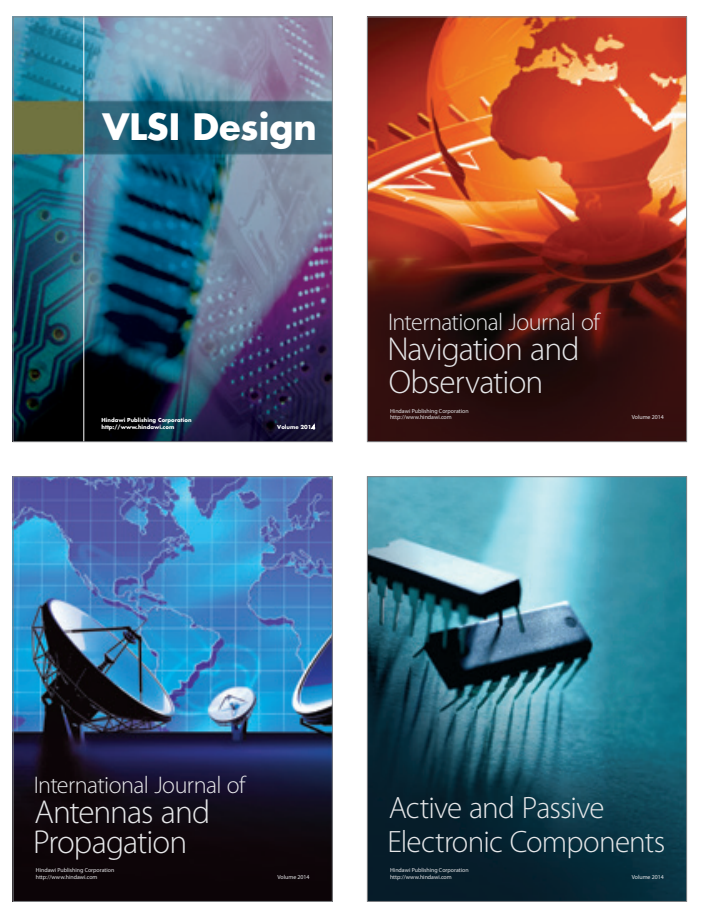
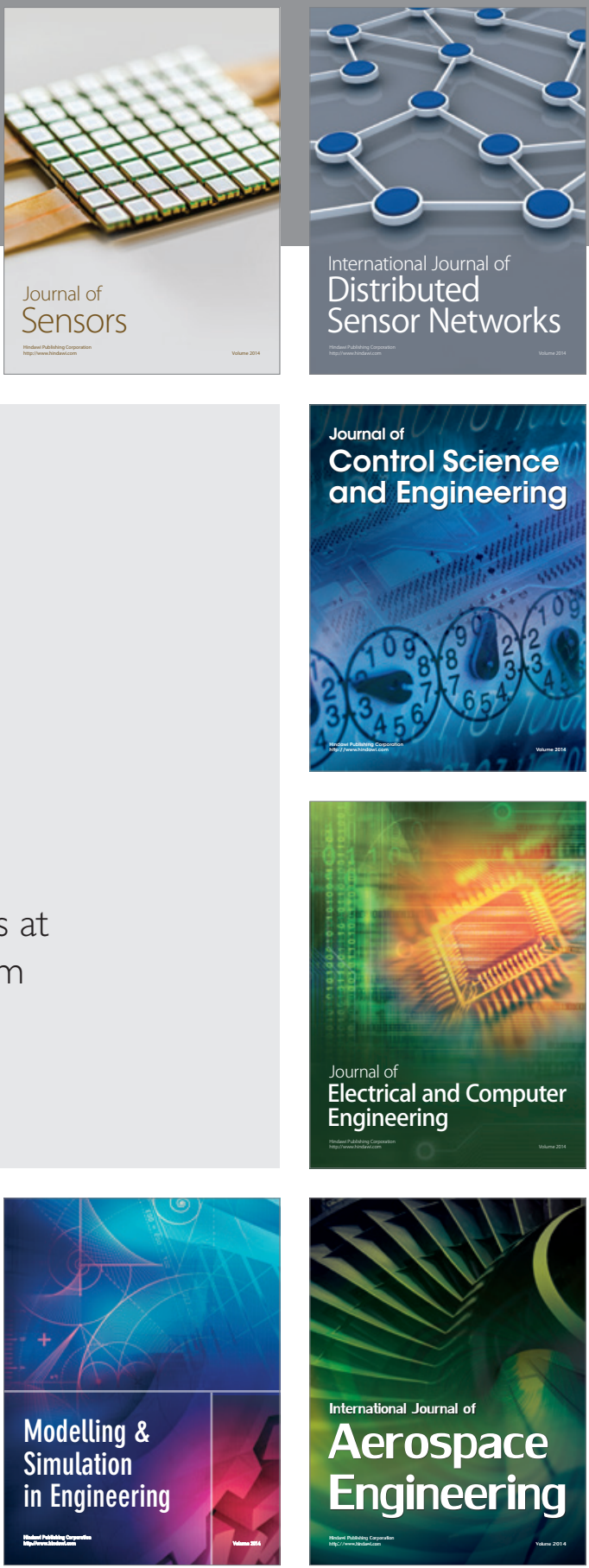

Journal of

Control Science

and Engineering
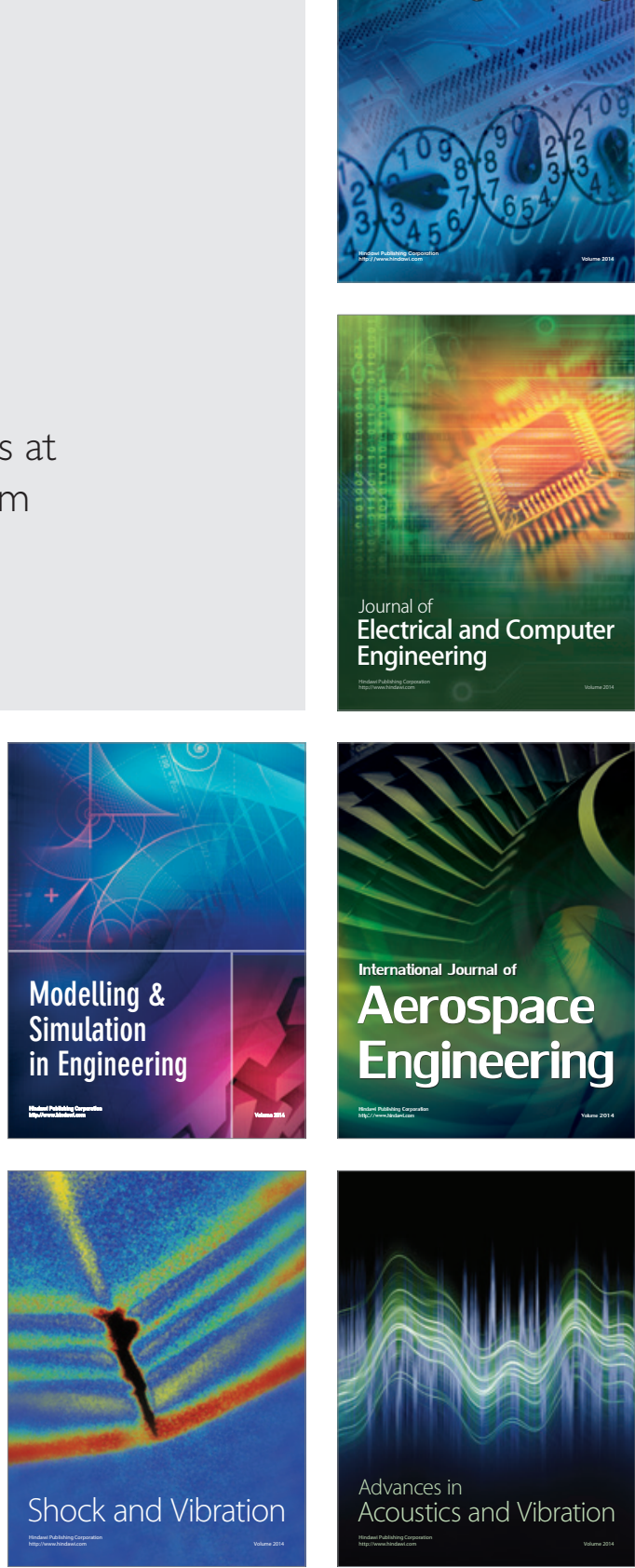